ACCEPTED MANUSCRIPT

\title{
Structural characterization of Au nano bipyramids: reshaping under thermal annealing, capping agent effect and surface decoration with $\mathrm{Pt}$
}

To cite this article before publication: María Fernanda Torresan et al 2019 Nanotechnology in press https://doi.org/10.1088/1361-6528/ab0144

\section{Manuscript version: Accepted Manuscript}

Accepted Manuscript is "the version of the article accepted for publication including all changes made as a result of the peer review process, and which may also include the addition to the article by IOP Publishing of a header, an article ID, a cover sheet and/or an 'Accepted

Manuscript' watermark, but excluding any other editing, typesetting or other changes made by IOP Publishing and/or its licensors"

This Accepted Manuscript is @ 2019 IOP Publishing Ltd.

During the embargo period (the 12 month period from the publication of the Version of Record of this article), the Accepted Manuscript is fully protected by copyright and cannot be reused or reposted elsewhere.

As the Version of Record of this article is going to be / has been published on a subscription basis, this Accepted Manuscript is available for reuse under a CC BY-NC-ND 3.0 licence after the 12 month embargo period.

After the embargo period, everyone is permitted to use copy and redistribute this article for non-commercial purposes only, provided that they adhere to all the terms of the licence https://creativecommons.org/licences/by-nc-nd/3.0

Although reasonable endeavours have been taken to obtain all necessary permissions from third parties to include their copyrighted content within this article, their full citation and copyright line may not be present in this Accepted Manuscript version. Before using any content from this article, please refer to the Version of Record on IOPscience once published for full citation and copyright details, as permissions will likely be required. All third party content is fully copyright protected, unless specifically stated otherwise in the figure caption in the Version of Record.

View the article online for updates and enhancements. 


\section{Article type: Full paper}

\section{Structural characterization of Au nano bipyramids: reshaping under thermal annealing, capping agent effect and surface decoration with $\mathbf{P t}$}

María Fernanda Torresan ${ }^{* 1,2}$, Paula C. Angelomé2, Lourdes Bazán-Díaz ${ }^{3}, J$. Jesús Velázquez-Salaza ${ }^{4}$, Rubén Mendoza-Cruz ${ }^{3}$, Rodrigo A. Iglesias ${ }^{1}$, Miguel José-Yacamán ${ }^{4}$

Dr. M. F. Torresan $* 1,2$

Dr. P. C. Angelomé ${ }^{2}$

Dr. L. Bazán-Díaz ${ }^{3}$

Dr. J. J. Velázquez-Salazar ${ }^{4}$

Dr. R. Mendoza-Cruz ${ }^{3}$

Dr. R. A. Iglesias ${ }^{1}$

Dr. M. José-Yacamán ${ }^{4}$

${ }^{1}$ Instituto de Investigaciones en Fisicoquímica de Córdoba, Universidad Nacional de Córdoba, INFIQC CONICET, Departamento de Fisicoquímica, Facultad de Ciencias Químicas, Pabellón Argentina, Ala 1 Piso 2, Ciudad Universitaria, Córdoba 5000, Argentina ${ }^{2}$ Gerencia Química - Centro Atómico Constituyentes, Comisión Nacional de Energía Atómica, CONICET, Av. Gral. Paz 1499, B1650KNA San Martín, Buenos Aires, Argentina.

${ }^{3}$ Department of Biomedical Engineering, The University of Texas at San Antonio, One UTSA Circle, San Antonio, TX 78249, United States of America.

${ }^{4}$ Department of Physics \& Astronomy, The University of Texas at San Antonio, One UTSA Circle, San Antonio, TX 78249, United States of America.

*Corresponding authors: fertorresan@gmail.com

Keywords: Gold nano bipyramids, thermal annealing, ligand exchange, Pt deposition

Abstract

Anisotropic gold nanoparticles offer potential applications due to the functionalities and the shape dependent properties. Reshaping noble metal nanoparticles is an interesting field with optical, Surface Enhanced Raman Spectroscopy (SERS), catalytic applications and potential application as a photothermic therapy. This work comprises the structural study on gold nanobipyramids (Au NBPs) and nanodumbbells (Au NDs) and the evolution of Au NBPs capped with Cetyl trimethylammonium bromide (CTAB) and dodecanethiol through an insitu and ex-situ heating process in high vacuum. Also, we study to reshape Au NBPs by the 
addition of Pt to study the surface modification and the strain generated on a single particle by geometric phase analysis (GPA).

\section{Introduction}

Metal nanostructures are an interesting group of materials whose properties are determined by their sizes and shapes. The considerable interest in studying gold nanostructures to be used in a variety of applications arise from the fact that gold has high electrical conductivity, reflectivity and constitutes a relatively inert material which provides stability and resistance to corrosion and oxidation to these nanomaterials. ${ }^{[1]}$ Also, nanostructured materials offer the opportunity to enhance their unique properties. For instance, noble metals nanoparticles (NPs), in particular Au NPs, possess strong plasmonic properties with potential applications. Localized surface plasmon resonance (LSPR) is an optical phenomenon generated by the collective oscillation of the electron gas in some metal nanostructures surrounded by a dielectric. In the case of Au NPs LSPR appear as a response to the absorption and scattering at specific resonant wavelengths when a gold NPs is irradiated with light and depends on the morphology and dielectric environment of the nanoparticle.. ${ }^{[2,3,4]}$ At present a wide variety of gold nanostructures with different shapes and sizes can be obtained by colloid chemistry, such as spherical nanoparticles, ${ }^{[5-7]}$ hollow nanoparticles ${ }^{[8]}$ and elongated structures as nanorods, ${ }^{[9-11]}$ nano bipyramids ${ }^{[10-12]}$ and nanowires. ${ }^{[13,14]}$ Nanorods and nano bipyramids (Au NBPs) exceed the spherical structures regarding the range of light absorption, since in the case of spherical structures the maximum absorption due to LSPR can occur between 500-600 nm, while the elongated structures can have absorption peaks in the near-infrared (NIR) region. ${ }^{[2]}$ This is important because of the so-called "biological transmission window" of $650-950 \mathrm{~nm}$, where tissues become transparent to electromagnetic radiation by applying NIR laser radiation for the excitation of the nanoparticles LSPR in Photothermal cancer theranostics. ${ }^{[2,15]}$ 
Notably, Au NBPs are gaining attention in the fields of sensing ${ }^{[16-19]}$ and catalysis. ${ }^{[20]}$ Also, the morphology of Au NBPs present sharp tips and edges and, in agreement with theoretical calculations, an essential local field enhancement is expected in those parts (hot spots). ${ }^{[16-21]}$ This makes them suitable candidates for surface-enhanced Raman spectroscopy (SERS). The synthetic methodologies to obtain Au NBPs are mainly based on the gold seed-mediated growth, kinetically assisted by the underpotential deposition of silver atoms. ${ }^{[11]}$ Afterward, the bipyramids can be regrown under different experimental conditions, mostly varying the relative amounts of surfactants, and the quantity of added silver, to obtain a different kind of Au NBP-based nanostructures such as larger bipyramids and dumbbells. ${ }^{[12]}$ The reshaping of the ends and size of those structures generates a finely tuning of the LSPR band in the visible and NIR region. ${ }^{[12]}$

There is plenty of work devoted to tailoring surface properties of Au nanostructures by attaching different types of organic molecules (known as "organic capping") and covering the surface with other metals or metal oxides. ${ }^{[23-28]}$

Capping molecules are usually surfactants or other organic molecules that are attached to $\mathrm{Au}$ surface and assist during the synthesis, keeping the nanoparticles stable and allowing their redispersion in different solvents, according to their polarity and nature. Commonly, Au NBPs are capped with Cetyl trimethylammonium bromide (CTAB) molecules. ${ }^{[11,12]}$ As some of the potential and future uses for these bipyramids may require high-temperature operation conditions, it is transcendental to study their thermal stability and to explore the role of capping agents in the thermal stability of these. Oxidative etching of Au bipyramids employing $\mathrm{H}_{2} \mathrm{O}_{2}$ or high temperatures and oxygen has been shown as a simple way to obtain Au nanorods of different morphologies using Au bipyramids as a starting point. ${ }^{[12]}$ This 
process reshapes bipyramids by cyclic oxidations of $\mathrm{Au}(0)$ to $\mathrm{Au}$ (III) caused by the oxidizing agents and deposition/redeposition of $\mathrm{Au}$ (III) to form Au back onto surface in lowenergy, and low- defect areas until the particles reaches an energy minimum, which is attributed to a gold nanorod. From these studies, information related to the thermal and chemical stability of these particles can also be extracted and inferred, but there is a lack of information regarding the influence of capping agents over this process. It was reported elsewhere ${ }^{[12]}$ that oxidative etching by heating over Au bipyramids capped with CTAB is slower in comparison with Benzyl dimethyl hexadecyl ammonium chloride (BDAC) capped bipyramids because of BDAC act as etchant together with the dissolved oxygen in solution.

Reshaping Au NBPs by surface modification with the deposition of other metals on their surface is an interesting case of study. For instance, decoration of the surface of $\mathrm{Au}$ nanostructures with metals can dramatically change the properties of the hybrid nanoparticle obtained. Those metals can form layers, $3 \mathrm{D}$ deposited structures or alloys. ${ }^{[20,29-35]}$ Also, it can be targeting photocatalytic properties with the deposition of different metals or metal oxides such as $\mathrm{Pt}, \mathrm{Pd}, \mathrm{Co}, \mathrm{ZnO}, \mathrm{TiO}_{2}$ and $\mathrm{Cu}_{2} \mathrm{O}$ in the synthesis of heterogeneous metallic particles. ${ }^{[36,20]}$ A selective growth of different materials such as $\mathrm{Ag}$, $\mathrm{Pt}$ or $\mathrm{SiO}_{2}$ over $\mathrm{Au} \mathrm{NPs}$ can be exploited as an alternative to change Au surface dependent properties, for example by tuning the plasmon resonance. ${ }^{[37]}$ In the present work, the surface modification of Au NBPs by using organic molecules and covering them with a catalytic metal was studied. In a first stage, the capping agent replacement was performed. Its effect over the thermal stability of Au NBPs was followed by in situ and $e x$-situ heating experiments under high vacuum conditions. The capping agent affects the surface energy of nanocrystals, influencing rearrangements of Au atoms under the heating process. Secondly, the surface modification was performed by the deposition of $\mathrm{Pt}$, in order to obtain bimetallic nanoparticles that present 
both, the Au plasmonic properties and Pt catalytic properties. The structural arrangement of Pt over Au NBPs surface was analyzed through atomic-resolution imaging. ${ }^{[20,31-33]}$

\section{Results and discussion}

\subsection{Reshaping of Au NBPs by thermal annealing}

The modification on the morphology and the surface reconstruction of Au NBPs can be optimized during the synthesis process. A fundamental step to obtain high yield of pure nanoparticles remains in the purification method, where the presence of CTAB or BDAC micelles, purify the final colloidal solution. The synthetic procedure employed previously reported ${ }^{[11,12]}$ yields highly reproducible Au NBPs batches. Two different types of Au NBPs were produced; Au regular bipyramids and Au nanodumbbells (Au NDBs). The main difference in the synthetic procedure to obtain both kinds of bipyramids are the employed surfactants. In the case of Au NBPs, only CTAB was employed while for the Au NDBs a mixture of CTAB/CTAC was used. CTAC preferentially covers the extremes of bipyramids, and the bonding strength of CTAC is weaker than with CTAB, which causes a preferential growth over the apexes. ${ }^{[12]}$

Figure 1 shows electron micrographs of the obtained Au seeds and the Au bipyramids, as well as their visible - NIR spectra. Au seeds of $\sim 5 \mathrm{~nm}$ in diameter presented the 5-fold decahedral structure, which eventually evolved into the elongated bipyramid morphology. Their absorbance spectrum showed the Surface Plasmon Resonance maximum at $508 \mathrm{~nm}$ which confirmed the small size of the nanoparticle seeds. Figure 1b) and Figure 1c) correspond to SEM images of Au NBPs and Au NDBs. The particle size increased from 100 $\mathrm{nm}$ to $150 \mathrm{~nm}$ (long-axis length), which is reflected on the red-shift of the plasmon resonance maximum. On the other hand, the typical widths of Au NBPs and Au NDBs were 27 and 37 
nm respectively. Both, Au NBPs and Au NDBs show the presence of the characteristic two peaks. The first one at $525 \mathrm{~nm}$ and $540 \mathrm{~nm}$ corresponding to the transverse plasmon resonance. The second maximum at $750 \mathrm{~nm}$ and $1000 \mathrm{~nm}$, for Au NBPs and Au NDBs, respectively, corresponds to the longitudinal plasmon resonance, being highly dependent on the aspect ratio of bipyramids.
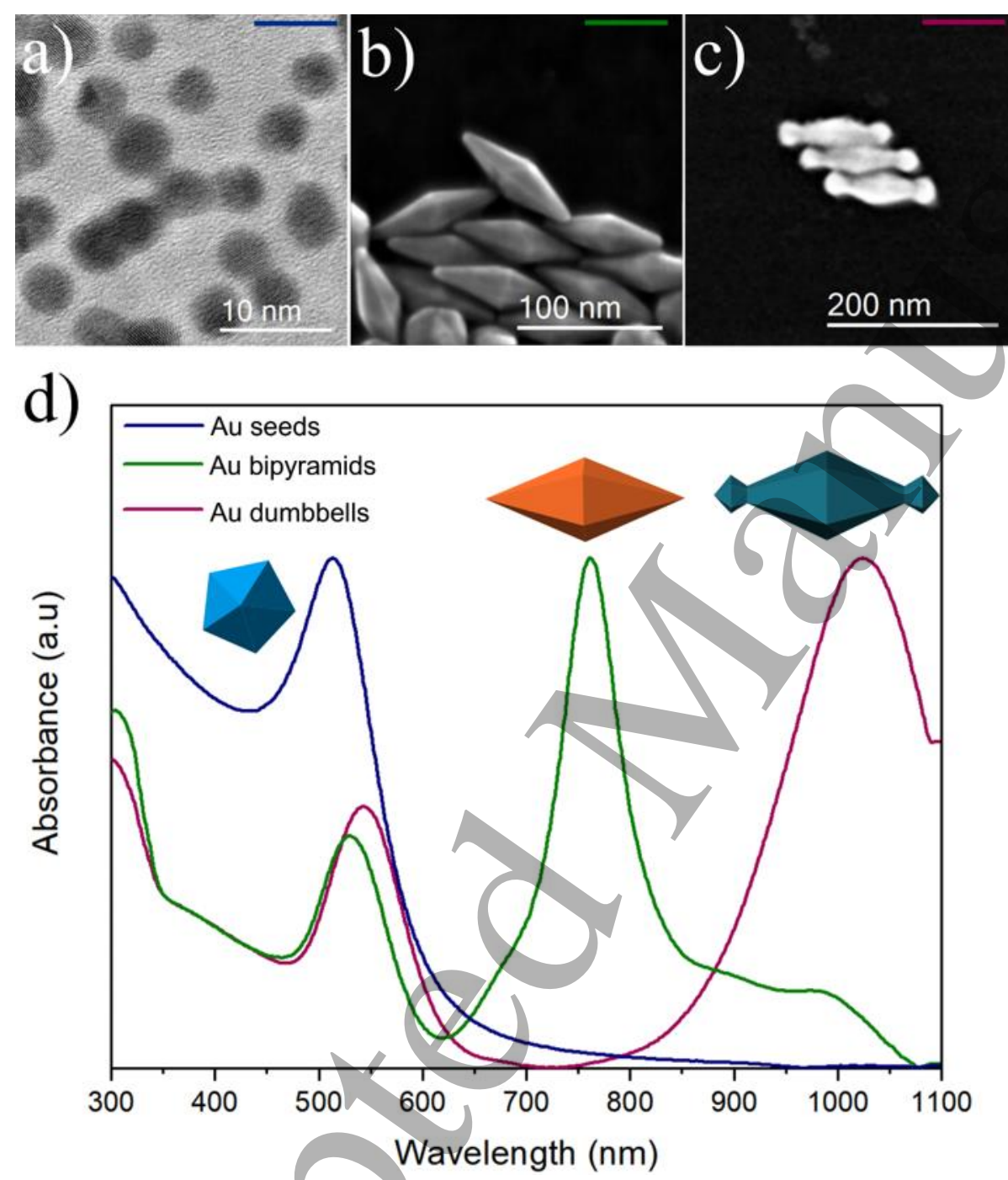

Figure 1. (a) TEM micrograph of Au seeds and (b, c) SEM micrographs of Au NBPs and Au NDBs respectively. (d) UV-Vis-NIR absorbance spectra of Au seeds and the obtained Au NBPs and Au NDBs.

The surface reconstruction and reshaping of nanoscale materials under thermal annealing is an interesting aspect to comprise because it is related to their thermodynamic stability. 
Additionally, the role of surface capping molecules in the behavior of these nanostructures under high temperature conditions arises as a corollary aspect to analyze. Au nanoparticles in different solvents subjected to heating showed an increase in their size due to coalescence or Ostwald ripening. ${ }^{[38,39]}$ Reshaping of Au triangular nanodisks to softer-edges and spherical nanoparticles was observed under heating, which was attributed to the lower melting point expected in nanoscale particles. ${ }^{[40]}$

A study of the thermal stability of Au NBPs and Au NDBs was performed by ex-situ and in situ heating experiments in high vacuum conditions. Figure 2 shows the effect of ex-situ heating experiments on bipyramids after 1 hour at $200^{\circ} \mathrm{C}$ and $400^{\circ} \mathrm{C}$. As-synthesized bipyramids present a five-fold symmetry along their long axis owing to the multi-twin nature of the seed particles. The five-fold symmetry is elucidated from the displayed electron diffraction pattern, showing the overlapping of the [112] and [001] patterns from three diffracting subunits. ${ }^{[41]}$ Hence, the bipyramids grown along the [110] direction of each decahedral seed, ending in sharp tips. The $\sim 16^{\circ}$ angle corresponding to the angle between the $2 \mathrm{D}$ projection of the edges and the five-fold axis, indicating that the faces correspond to highindex facets. Considering a truncated bipyramidal shape as model with $86 \mathrm{~nm}$ in length and $30 \mathrm{~nm}$ width, the expected angle between the five-fold axis and each facet is $\sim 13.5^{\circ}$. This angle can be related to $\{116\}$ high-index planes, the high-energy planes forming each facet of the bipyramids.

As warming takes place, bipyramids start to lose their morphology as edges start to smooth. After 1 hour of heating at $200{ }^{\circ} \mathrm{C}$, bipyramids were converted to oval structures with a decrease in the length from $86 \mathrm{~nm}$ to $60 \mathrm{~nm}$ in comparison with the dimensions of the bipyramids before heating and an almost imperceptible change in the width (Figure 2). The elongated shape still remained. However, as shown in Figure 2b, the top edges became long $\{220\}$ edges, and the tips presented two reconstructed facets, with approximated angles of $59^{\circ}$ and $34^{\circ}$ respect to the [110] five-fold axis. Taking into consideration the same analysis for a 
truncated bipyramidal shape, the facets should form approximated angles of $53.5^{\circ}$ and $28.7^{\circ}$, respectively. These angles were correlated with $\{111\}$ and $\{2-25\}$ facets, which form calculated angles of $54.5^{\circ}$ and $29.3^{\circ}$, respectively. All these facets represent lower energy facets compared to the original $\{116\}$ higher-index facets. ${ }^{[42,43]}$ After 1 hour of heating at $400{ }^{\circ} \mathrm{C}$, it can be noticed a more isotropic and spherical structure, following the trend of decreasing the length to $55 \mathrm{~nm}$ (approx. 30-40\% decrease in comparison with the nonannealed Au NBPs) and increasing the width to $45 \mathrm{~nm}$ (approx. 50\% increase in comparison with the non-annealed Au NBPs).

In the case of Au NDBs, the trends were similar, with a decrease of 30-40\% in length after annealing at $400{ }^{\circ} \mathrm{C}$. The Au NDB in Figure 2d taken along the [111]/[110] zone axis showed a $14^{\circ}$ side, corresponding to $\{116\}$ facets. The same angle was formed with the broad tip. After annealing at $200^{\circ} \mathrm{C}$, the particle underwent a shape transition exposing $\{220\}$ edges at the top, and slightly shaper tips compared to the previous case. However, after annealing at $400^{\circ} \mathrm{C}$ the particle retained its elongated shape, exposing longer $\{220\}$ edges and shorter tips. From this analysis, it is inferred that both bipyramidal and dumbbell morphology correspond to a thermodynamically metastable structure, undergoing a shape transition from high-index facets to low-energy facets, and finally to a quasi-spherical morphology after annealing. Because of the high surface area exposed by nanoparticles, the spherical shape, with minute facets, represents a minimum energy configuration that lowers the surface energy for both types of bipyramids. ${ }^{[44]}$ This is a consequence of the trending to decrease the surface area at the same volume, to lower the overall surface energy. From the SAED patterns, it is clear that the 5-fold symmetry of the nanoparticles after thermal annealing remained indicating that the melting point was not reached. The small particles that appear around the Au bipyramids while heating can be a consequence of the thermally assisted reduction of remains of $\mathrm{HAuCl}_{4}$. 


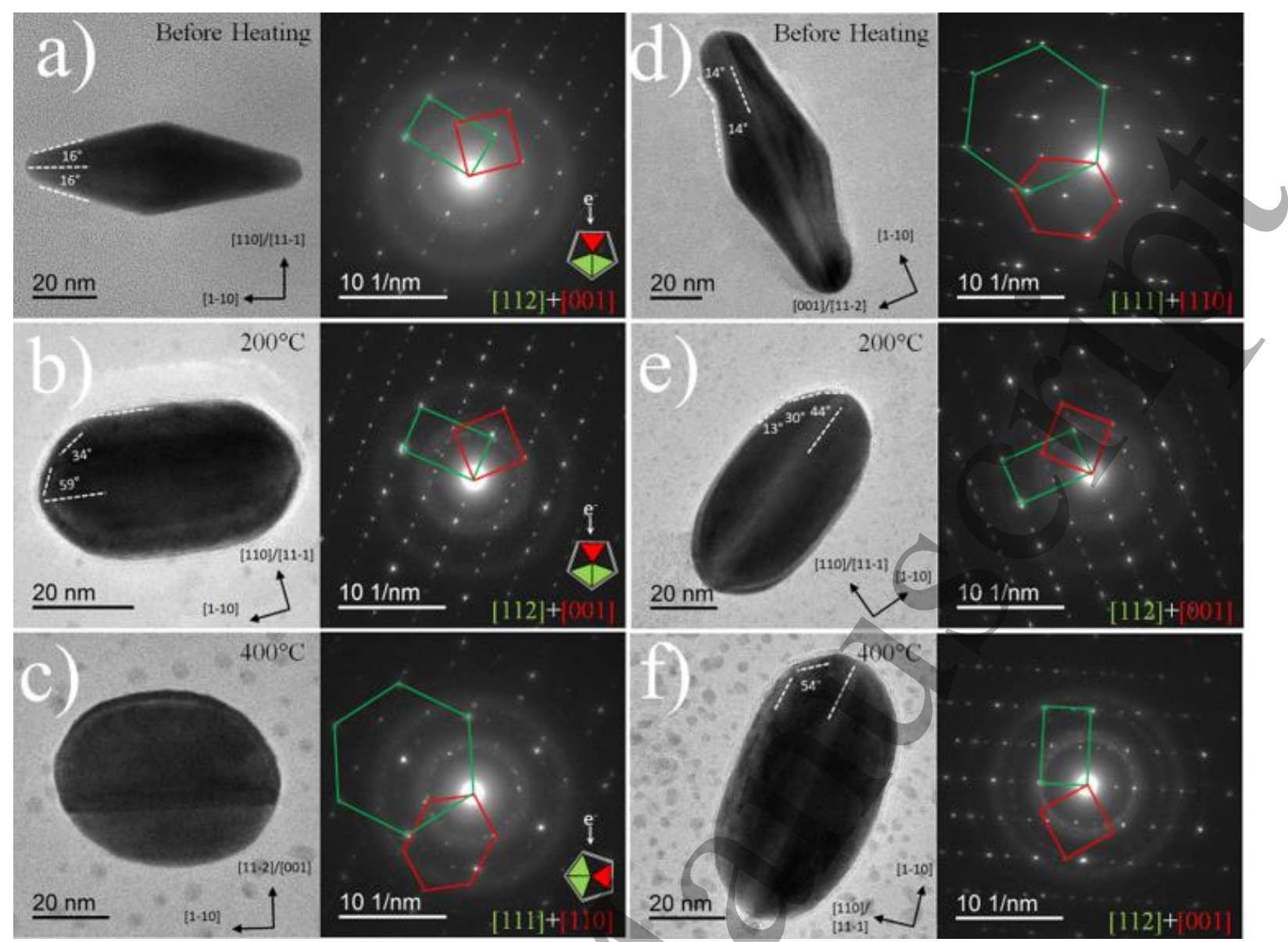

Figure 2. TEM images and SAED patterns of the Au plane-bipyramids (a-c) and dumbbell bipyramids (d-f), before and after heating annealing in vacuum for $1 \mathrm{~h}$ at different temperatures. The SAED patterns correspond to the projected patterns of the individual monocrystalline units. Geometrical models of the beam direction over the nanoparticles are shown as insets. After $200^{\circ} \mathrm{C}$, both type of particles underwent a marked reshaping. However, the 5-fold symmetry was retained, indicating that atomic distribution takes place to lower the surface energy without reaching melting of the particle.

The oxidation of gold bipyramids employing $\mathrm{H}_{2} \mathrm{O}_{2}{ }^{[45]}$ or an oxidative etching with the oxygen dissolved on the surrounding media, while heating a $120^{\circ} \mathrm{C}^{[12]}$ cause in both cases a similar change in the bipyramid's morphology. In the first case, it was reported that oxidation takes place preferentially at surface sites with a high curvature such as apexes and edges and that both, the length and the width of bipyramids decrease as bipyramids tend to sphere, with the concomitant appearance of an absorption signal of dissolved Au (III). In our study, the length of bipyramids is shortened but the width is increased, so this is not a case of oxidation and loss of $\mathrm{Au}$ atoms as in oxidative etching cases that are known to reshape crystals via atomic addition and subtraction. ${ }^{[46]}$ 
The influence of capping molecules or stabilizing agents on the thermal stability of $\mathrm{Au}$ bipyramids was analyzed. In the case of the obtained bipyramids, the surfactant that they have after the synthesis is CTAB. It is reported that CTAB is attached to the surface via an $\mathrm{Au}-\mathrm{Br}$ bond, that can be detected from a characteristic signal at $190 \mathrm{~cm}^{-1}{ }^{[47]}$ of the Raman spectrum, as can be extracted from Figure 3. A procedure to replace CTAB molecules for dodecanethiol molecules was carried out. Although this ligand exchange was reported for nanorods ${ }^{[48]}$, we could adapt the process for Au NBPs in the present work. The successful replacement of CTAB capping molecules was demonstrated by Raman spectroscopy, following the disappearance of the Au-Br signal of CTAB capped particles and the appearance of the Au-S signal near $250 \mathrm{~cm}^{-1}{ }^{[47,49]}$ for dodecanethiol capped ones (Figure 3).

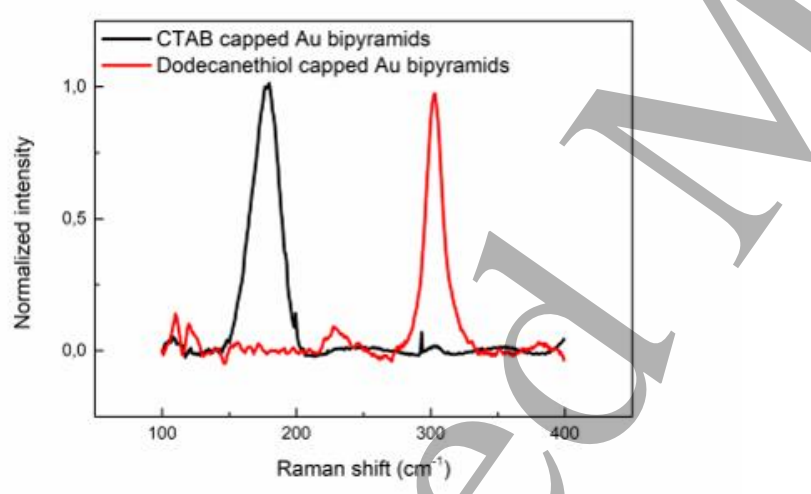

Figure 3. Raman spectra of Au NBPs capped with CTAB (black line) and dodecanethiol (red line).

HAADF-STEM images of bipyramids taken in situ while heating at $94{ }^{\circ} \mathrm{C}$ and the change in the dimensions measurements over time are presented in Figure 4 in the case of Au NBPs with CTAB and in Figure 5 for bipyramids with dodecanethiol. The chosen temperature to analyze the stability of the particles was slightly below $100^{\circ} \mathrm{C}$ as one on the potential uses for these nanoparticles is related to nanotheranostic and photothermal therapy applications, where the optimum temperature range lies between 37 and $60{ }^{\circ} \mathrm{C} .{ }^{[50,51]}$ It is worth to mention that 
the chose temperature is far below from the desorption temperatures of both capping agents.

In the case CTAB-capped Au nanorods show three different desorption temperatures at $230{ }^{\circ} \mathrm{C}$ due to the evaporation of weakly bounded surfactant monomers, $273^{\circ} \mathrm{C}$ and $344{ }^{\circ} \mathrm{C}$ attributed to the outer and inner layer of the adsorbed membrane. ${ }^{[52]}$ On the other hand, in the case of dodecanethiol bounded to a gold surface, the desorption temperature found in literature was between 230 and $330{ }^{\circ} \mathrm{C} .{ }^{[53]}$

a)

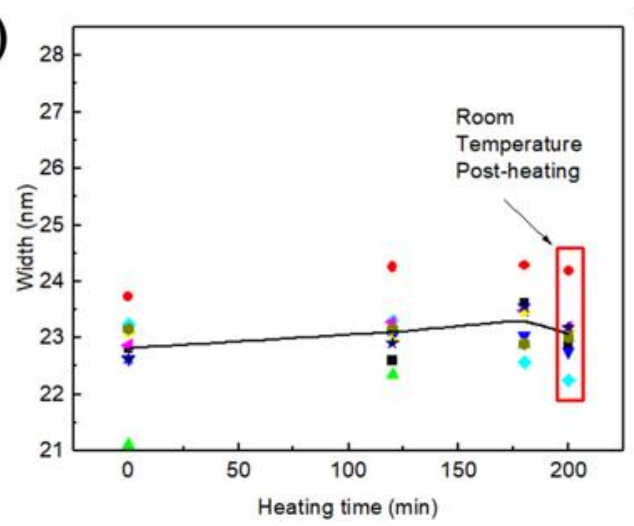

b)

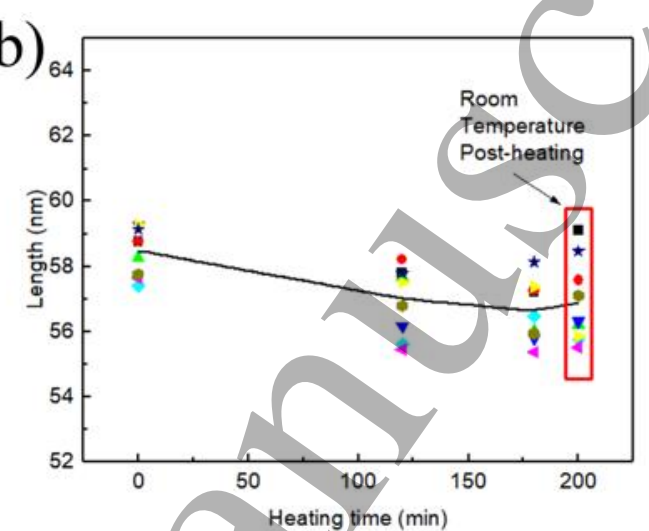

d)
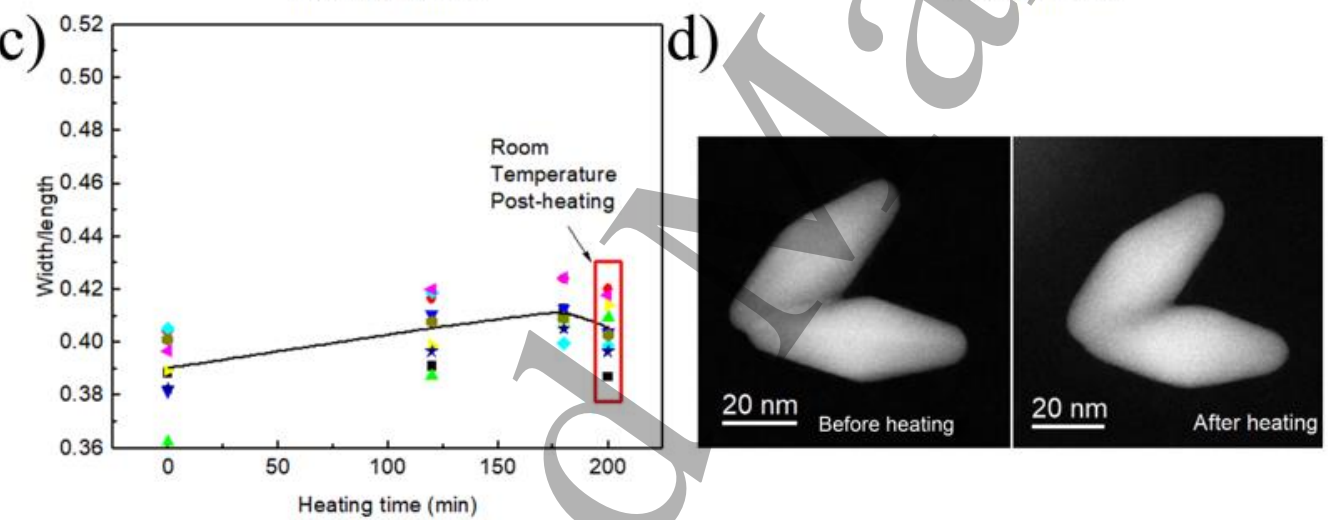

Figure 4. Changes in the dimensions ((a) width, (b) length and (c) width/length ratio) of CTAB capped Au bipyramids while heating. Each color corresponds to different particles. (d) STEM micrographs of two bipyramids before and after heating. 
a)
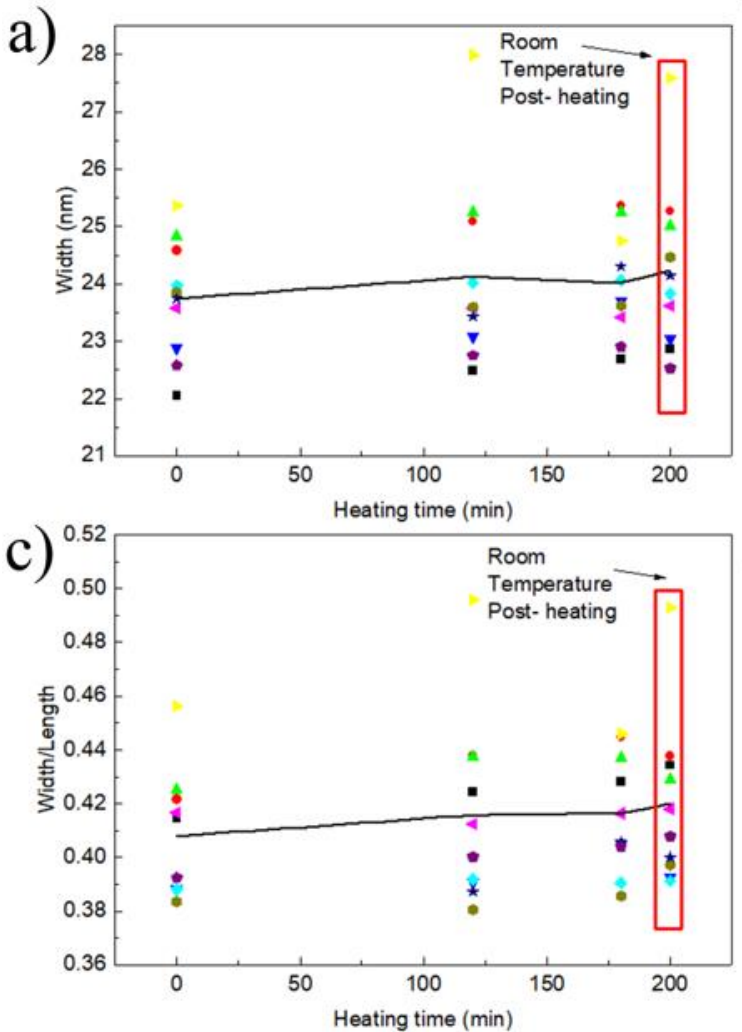

b)

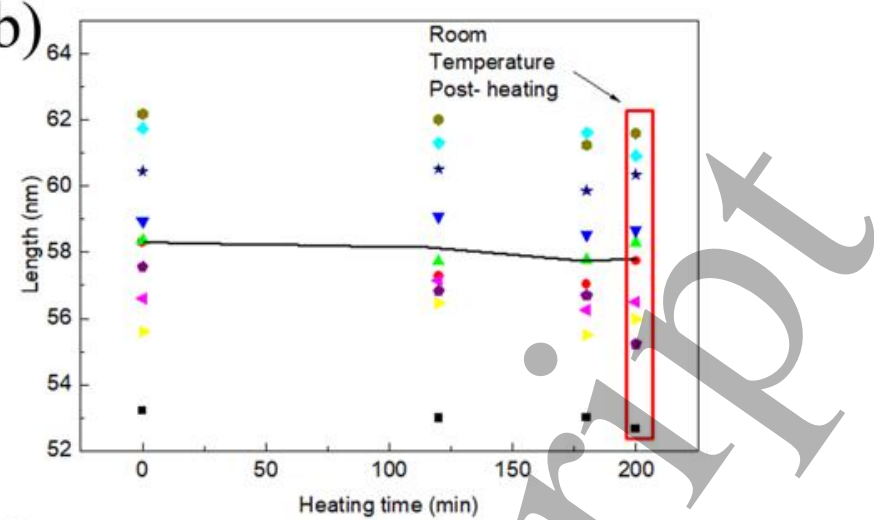

d)

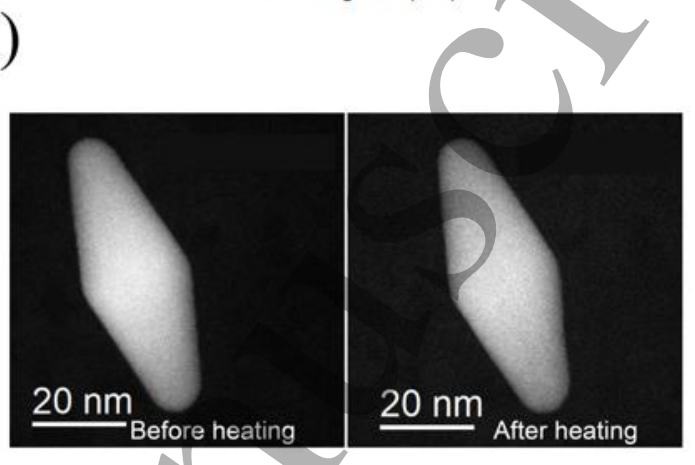

Figure 5. Changes in the dimensions ((a) width, (b) length and (c) width/length ratio) of dodecanethiol capped Au bipyramids while héating. Each color corresponds to different particles. (d) STEM micrographs of bipyramids before and after heating.

First, it can be observed that, for CTAB case, the change in the morphology of Au bipyramids is less marked at $94^{\circ} \mathrm{C}$ in comparison with the changes observed at higher temperatures (Figure 2). However, by measuring the dimensions of the bipyramids after different times of heating at $94{ }^{\circ} \mathrm{C}$, it can be noticed that there is a trend of a decrease in the length and an increase in the width over the heating time, even at the low temperature $94^{\circ} \mathrm{C}$. As a control, in each case one of the bipyramids was irradiated with the electron beam all the time during heating (See Figure S1 and Figure S2 of Supporting Information), and the trend was the same as for the other bipyramids that were only heated, not irradiated. These results indicate that the observed features depend exclusively over the temperature. This type of lattice contraction where the contraction along one crystallographic direction is usually accompanied by expansion along the others is common in the case of anisotropic systems, and it is referred as negative thermal expansion. ${ }^{[54]}$ An interesting fact arises when measuring the dimensions 
of the bipyramids when they reached room temperature after being heated for 3 hours at $94{ }^{\circ} \mathrm{C}$. It can be noticed a little throwback in the trend, meaning that there is a slight increase in the length and a decrease in the width of bipyramids when they return to be at room temperature. This effect can be related to a grade of elastic nature deformation capacity of gold bipyramids, in other words, a "memory" effect. From a more in-depth study of this effect, mechanical properties of this kind of nanoparticles can be inferred. Also, the whole trend is more marked in the case of bipyramids with CTAB in comparison with the ones stabilized with dodecanethiol. This effect is related to the bonding energy on the bipyramids, a strong bond has been found for $\mathrm{Au}-\mathrm{S}(40 \mathrm{kcal} / \mathrm{mol})^{[54,55]}$ in comparison with the Au-CTAB bond so the mobility of Au surface atoms is reduced allowing a favorable and higher stability on the crystalline structure of bipyramids with dodecanethiol.

\subsection{The decoration of Au NBPs with Pt nanostructures.}

Another approach to surface modification of Au bipyramids is the deposition of Pt over the nanoparticles surface. Mixing Au bipyramids plasmonic properties with the catalytic activity of other noble metals is an interesting option for several applications that require both features. Thus, to enhance the catalytic activity of metal nanoparticles, the synthesis of nanosized bimetallic particles must be achieved. Depending on the synthetic method and on the nature of the metals that are mixed, core-shell systems or homogeneous alloys can be obtained, ${ }^{[35]}$ also aggregated phases can be obtained. ${ }^{[56]}$

Figure 6 (a-d), shows SEM micrographs of Au bipyramids decorated with Pt, employing Pt solutions of different concentrations. When the concentration of $\mathrm{HPtCl}_{6}$ in the reaction solution is low, $\mathrm{Pt}$ forms deposited aggregates over the edges of Au bipyramids. As Pt concentration increases, the formed aggregates start to be located relatively uninformed over the whole surface of Au. It is reported that the growth of thin films of Pt over Au obeys the 
Stranski-Krastanov model for heterogeneous nucleation and growth theory. ${ }^{[57]}$ This model represents a growth type in which the metal tends to form 2D islands over the substrate, and afterward, these islands can grow in a 3D way, generating particles. The growth mode is mainly determined by the lattice match and interactions between the overlayer and substrate thus Stranski-Krastanov model ${ }^{[58]}$ is an intermediate mode between layered growth (Frankvan der Merwe) ${ }^{[59]}$ and the island growth (Volmer-Weber) ${ }^{[60]}$. Also, the "wettability" of the substrate surface with the shell material, related to the relative electronegativity of both metals, is determinant in the growth model of the system. ${ }^{[29]}$
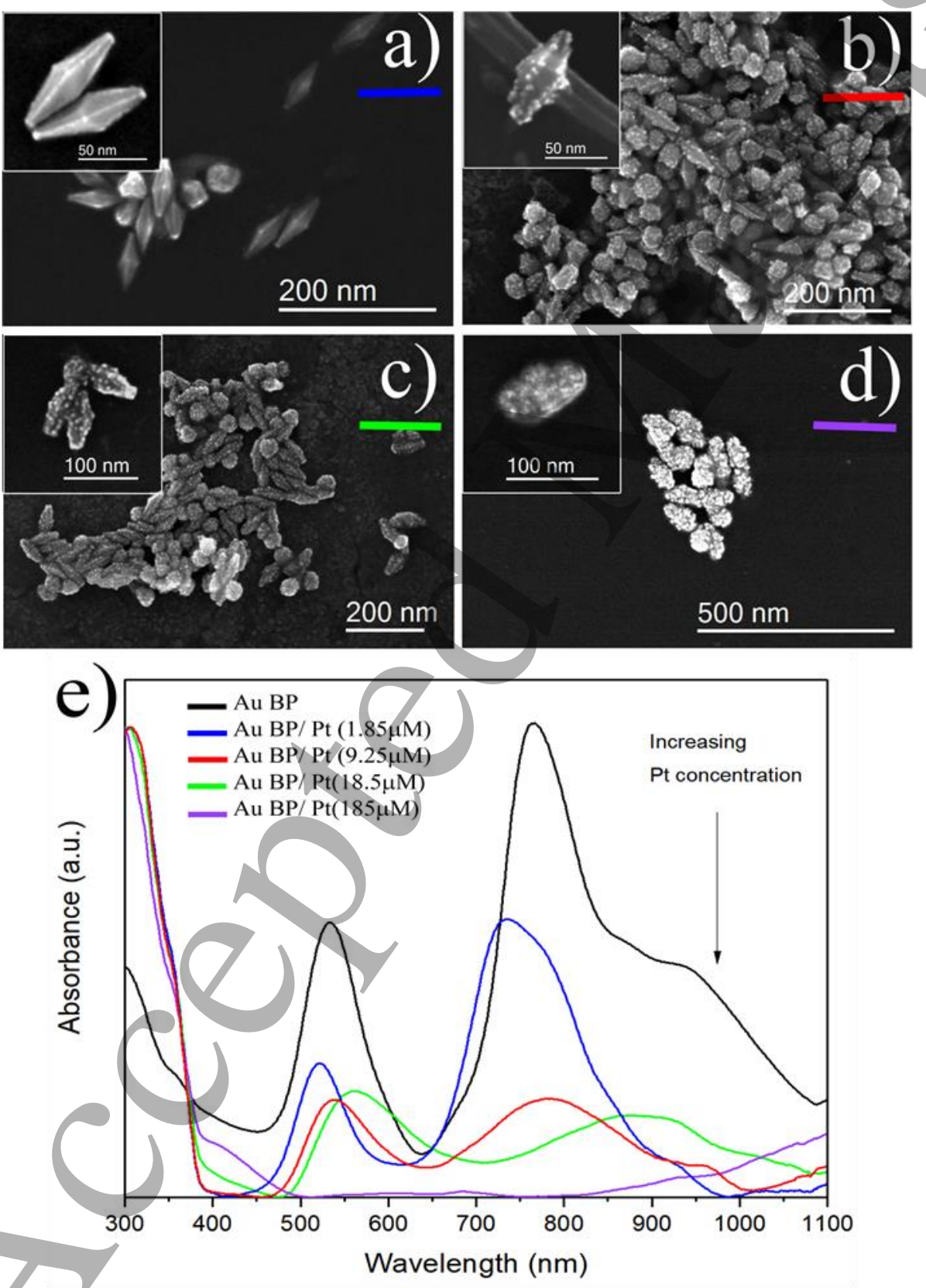
Figure 6. SEM micrographs Pt-decorated Au-bipyramids employing different concentrations of Pt a) $\left.1.85 \times 10^{-6} \mathrm{M}, \mathrm{b}\right) 9.25 \times 10^{-6} \mathrm{M}$, c) $1.85 \times 10^{-5} \mathrm{M}$ and d) $1.85 \times 10^{-4} \mathrm{M}$. e) The absorption spectra of the corresponding samples.

As the Au surface coverage degree with Pt increases, the surface plasmon signal decreases (Figure 6e), because less amount of $\mathrm{Au}$ is present on the surface. Thus, the potential enhancement of the catalytic properties of these combined particles can be detrimental to applications that require the presence of the surface plasmon absorption band. ${ }^{[31]}$ Also a red shift of the plasmon signals is observed when increasing the amounts of Pt deposited over $\mathrm{Au}$ NBPs. It has been reported that longitudinal surface plasmon resonance in non-spherical Au particles is very sensitive not only to slight morphological changes but also to atomic level roughness due to crystallographic reconstructions. ${ }^{[61]}$ Therefore, the observed red shift of the plasmon signals can be attributed to lattice reconstruction or an increase of Au NBPs surface roughness as a consequence of the Pt deposition process. Among the obtained samples, the one that retains a notable plasmon resonance signal and that presented uniformly sized and distributed Pt aggregates was selected to be fully characterized. The high angle annular dark field (HAADF) images and energy-dispersive spéctroscopy elemental mapping images are shown in Figure 7.

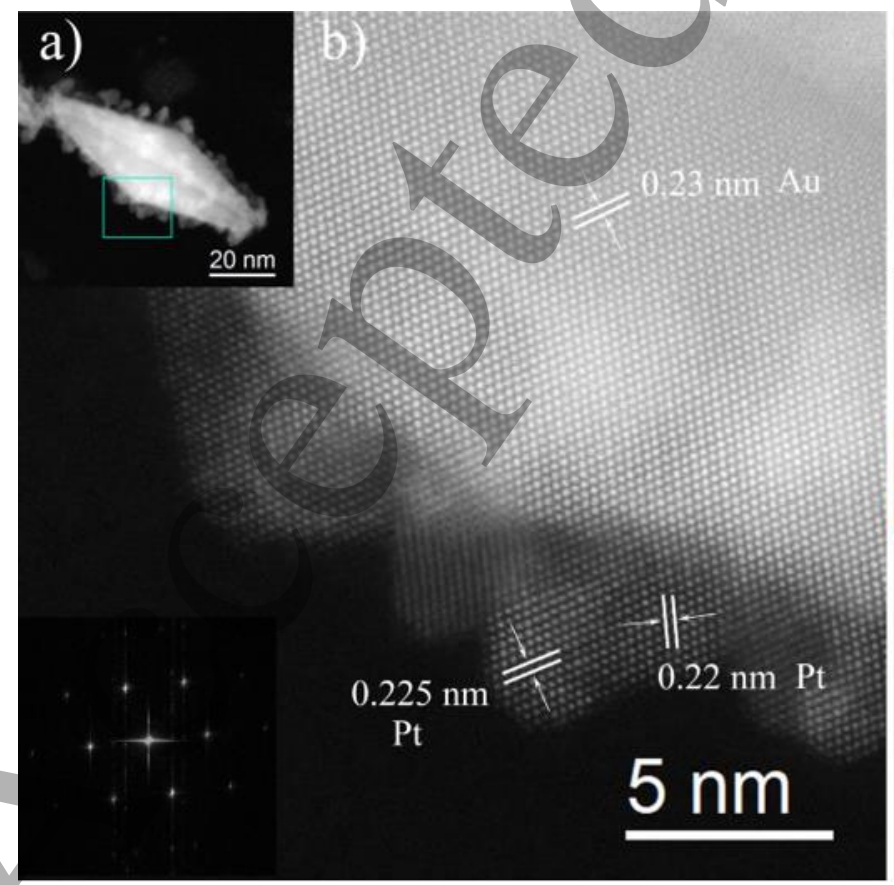

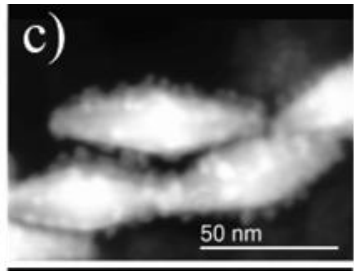
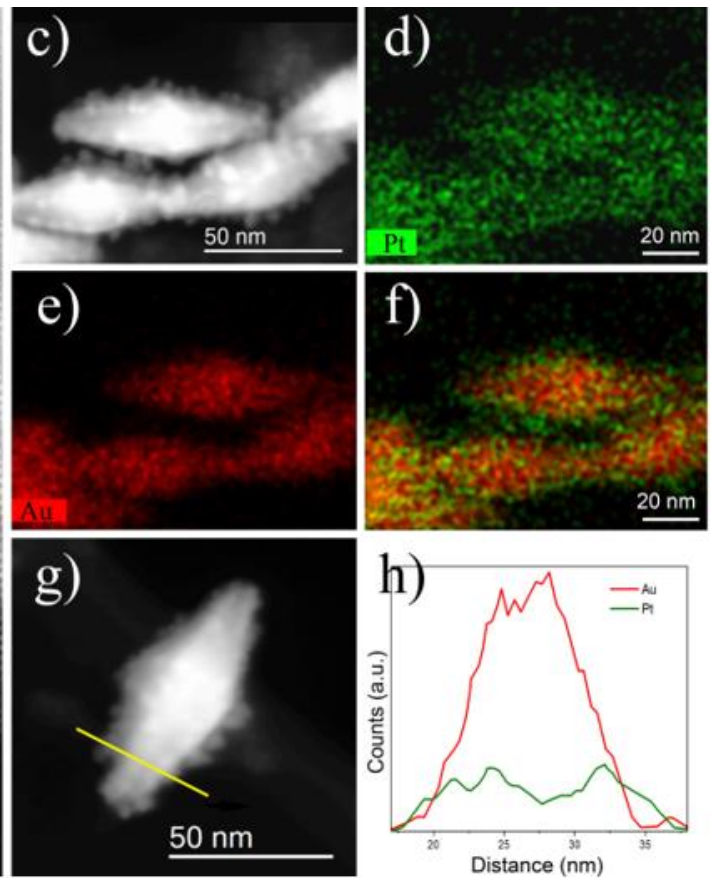
Figure 7. (a) STEM low magnification image of Pt decorated Au NBP. (b) Highmagnification STEM image of the square region in (a), (c-f) STEM-EDS elemental mapping images of $\mathrm{Au} / \mathrm{Pt}$ bipyramids, (g-h) STEM image and cross-sectional compositional line profiles of an $\mathrm{Au} / \mathrm{Pt}$.

Also Figure 7 shows a low and high magnification STEM image of an Au-Pt pyramid (Figure 7a) and Figure 7b) correspondingly). Additional HAADF analysis showed how the Pt particles tend to deposit over the edges of bipyramids. Some of the Pt nanodeposits are faceted. Fast-Fourier transform (FFT) power spectrum analysis shows that both nanostructures are oriented along the [011] zone axes. The distance between Au-Au peaks is $0.23 \mathrm{~nm}$, and Pt-Pt peaks are 0.225 . These values are consistent with the ones corresponding to $\mathrm{Au}\{111\}$ and $\mathrm{Pt}\{110\}$ planes respectively. The elemental map of the Au-Pt nanostructures was obtained using energy dispersive spectroscopy (Figure 7c) Figure 7d) and Figure 7e)). Different colors indicate the presence of different elements, where green and red refer to the presence of Pt and Au respectively. Moreover, the overlap of both elements is shown in Figure 7f). Figure 7g) and Figure 7h) shows a line-scan EDS spectrum of Au and Pt through a yellow crossing line in an individual nanoparticle. The Au signal was obtained only across the core region, whereas the Pt signal was traced across the entire particle, especially in the edges of the bipyramid.

The geometrical-phase analysis (GPA) method was used to quantify the strain distribution field relaxation map in Au-Pt. To perform GPA, Fourier filtering and image processing are used in high resolution electron microscopy images to improve the contrast, locate lattice mismatch sites and calculate the displacement and strain field mappings in a crystal lattice. ${ }^{[62]}$ This is achieved by placing masks around the different Bragg spots in the Fourier transform images and then forming the image by applying the inverse Fourier transform. From the image formed by the strong lattice reflections, it becomes possible to determine local variations in the structures, which is especially useful in nanomaterials with interfaces between two components. ${ }^{[62]}$ 
The Au/Pt interface was studied through GPA. Figure 8 a) shows a low magnification HAADF-STEM image of a gold bipyramid that was decorated with Pt. Figure 8 b) corresponds to a magnified area of one facet oriented along the [110] axis zone. The (11-1) and (-11-1) spots were chosen for GPA, the Gaussian mask was placed around these spots to isolate them, and the strain maps were calculated. It can be distinguished high strain is accumulated at the interface between $\mathrm{Au}$ and $\mathrm{Pt}$ originated by the lattice mismatch between both metals (Figure $8 \mathbf{c}$ )). The strain values are represented by a temperature scale, going from $10 \%$ tension strain to $-10 \%$ compression strain. The intensity profile across the atomic columns, Figure 8 d), indicated that strain was approximately constant along the $\mathrm{Au}$ bipyramid and abruptaly changed to $\sim 4 \%$ compressional strain at the interface with Pt. The magnified HAADF-STEM image and the intensity line profile on it showed the atomic displacement at the Au-Pt interface as a consequence of the accumulative lattice strain (Figure 8 e)). This indicated that stacking faults were formed and accumulated during the growth of the lattice-mismatched shell.
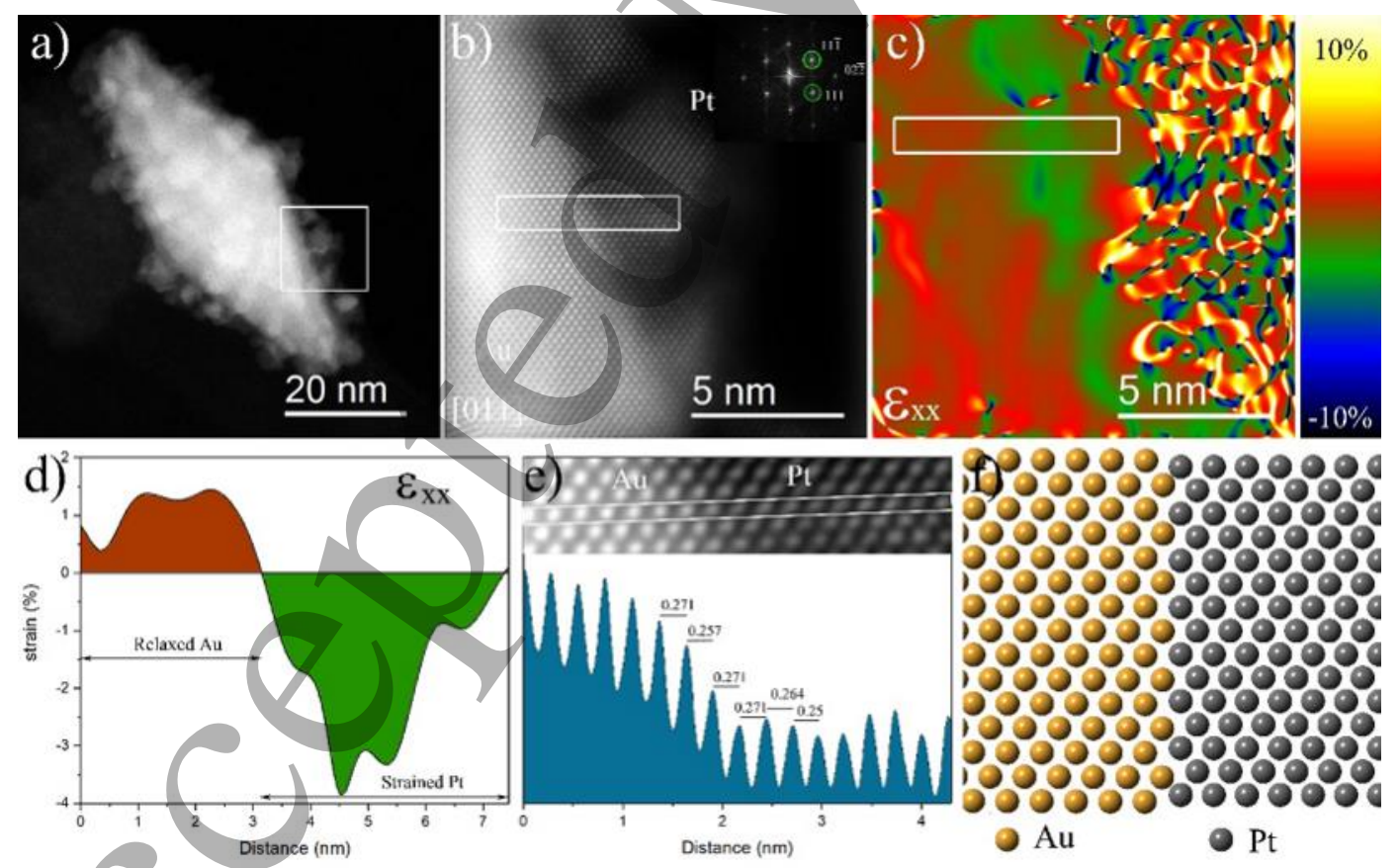

Figure 8. a) Low magnification HAADF-STEM image of a Pt decorated Au NBP and (b) magnified image of the square region in (a). The green circles in the FFT indicate (11-1) and $(-11-1)$ spots lattice fringes. (c) Strain distribution $\left(\varepsilon_{\mathrm{xx}}\right) \underline{\text { at }}$ the interface obtained by GPA. (d) Strain linear profiles showing the lattice displacement of around $1.5 \%$ within $\mathrm{Au}$, and $3.8 \%$ 
within Pt. (e) Magnified atomic-resolution image of the square region (b) and its intensity profile showing the atomic displacement at the interface of Au and Pt. (f) Diagram of the atomic distribution in the interface between $\mathrm{Au}$ and $\mathrm{Pt}$.

\section{Conclusion}

$\mathrm{Au}$ NBPs represent a versatile platform for a wide range of applications tuning their properties by surface functionalization either by capping exchange procedures or metal surface deposition. Thermal annealing of the bipyramids caused a drastic morphology change attributed to the minimization of the surface energy. CTAB capping molecules can be easily replaced by dodecanethiol, and it was detected a difference in the behavior when performing thermal annealing. The changes in dimensions while heating CTAB capped particles is more prominent in comparison with dodecanethiol capped particles due to the stronger interaction between thiol molecules and Au surfaces. It can be noticed a little throwback in the deformation trend when Au NBPs return to be at room temperature after being annealed at $94^{\circ} \mathrm{C}$. It is interesting to explore this effect as it is related to a grade of elastic nature deformation capacity of Au NBPs.

The surface modification of Au bipyramids with Pt post-growth nanostructures was also studied as a possibility to combine plasmonic properties of Au bipyramids with Pt catalytic properties. Pt was deposited onto the particles, forming agglomerates over the Au surface, especially over edges, obeying the Stranski Kastanov epitaxy growth model. The amount of deposited Pt can be controlled by the synthetic conditions, which also determines the intensity of the Au plasmon band. Thus, to satisfactorily combine the catalytic and plasmonic properties of both metals, a balance between the amount of Pt and Au in the surface and a strain engineering should be targeted.

4. Experimental Section 
Reagents: All the following reagents were purchased from Sigma Aldrich and used without further purification. Tetrachloroauric acid $\left(\mathrm{HAuCl}_{4} \cdot 3 \mathrm{H}_{2} \mathrm{O}, \geq 99.9 \%\right)$, Sodium borohydride $\left(\mathrm{NaBH}_{4}\right)$, citric acid trisodium salt dihydrate $(\geq 99.9 \%)$, Cetyl trimethylammonium bromide (CTAB, Bioxtra, 99\%), Cetyl trimethylammonium chloride (CTAC, $\geq 98 \%$ ), Benzyl dimethylhexadecylammonium chloride (BDAC), L-ascorbic Acid (AA, Bioxtra, 98\%), Silver nitrate $\left(\mathrm{AgNO}_{3}\right.$, BioXtra, >99\%), 1-dodecanethiol and Hexachloroplatinic (IV) acid hydrate $\left(\mathrm{H}_{2} \mathrm{PtCl}_{6}, \geq 99.9 \%\right)$. Hydrochloric acid solution $(\mathrm{HCl}, 1 \mathrm{~N})$ was purchased from Fisher Scientific. Also, deionized water was used in all the experiments.

Synthesis of Au NBPs: The Au NBPs were synthesized according to the previous reported methods with some modifications. ${ }^{[10,11]}$ Briefly, to produce Au bipyramids, the first step was the synthesis of Au seeds by mixing ultrapure water $(18.95 \mathrm{ml}), \mathrm{HAuCl}_{4}(0.25 \mathrm{~mL}, 10 \mathrm{mM})$ and sodium citrate $(0.5 \mathrm{~mL}, 10 \mathrm{mM})$, followed by a rapid addition of cold and fresh $\mathrm{NaBH}_{4}$ $(0.3 \mathrm{~mL}, 10 \mathrm{mM})$. This solution was kept under magnetic stirring at room temperature for two hours. Then, the plain bipyramids were grown in a solution containing CTAB (10 mL, $0.1 \mathrm{M})$, $\mathrm{HAuCl}_{4}(0.5 \mathrm{~mL}, 0.01 \mathrm{M}), \mathrm{AgNO}_{3}(0.1 \mathrm{~mL}, 0.01 \mathrm{M}), \mathrm{HCl}(0.2 \mathrm{~mL}, 1 \mathrm{~N})$, ascorbic acid $(0.08$ $\mathrm{mL}, 0.1 \mathrm{M})$, and the prepared $\mathrm{Au}$ seed solution $(95 \mu \mathrm{L})$. The mixture was stirred and kept in an oil bath at $30{ }^{\circ} \mathrm{C}$ for two hours. The growth solution was centrifuged at $11000 \mathrm{rpm}$ for $15 \mathrm{~min}$ and washed twice with CTAB $(10 \mathrm{~mL}, 1 \mathrm{mM})$. The obtained precipitate was dispersed in CTAB (3 mL, $1 \mathrm{mM})$ for further purification. First, to produce the selective precipitation of the pyramids separated from the seeds, three ml of CTAB solution was mixed with BDAC solution $(6.2 \mathrm{~mL}, 0.5 \mathrm{M})$ and of ultrapure water $(0.8 \mathrm{~mL})$ and left in an incubator at $37^{\circ} \mathrm{C}$ overnight. The supernatant was removed, and CTAB $(3 \mathrm{~mL}, 1 \mathrm{mM})$ was added to the vial to disperse the precipitate helped with an ultrasonic bath. The resulting purified solution was centrifuged at $10400 \mathrm{rpm}$ for $8 \mathrm{~min}$ and washed twice with CTAB (1 mL, 1mM). Finally, the purified bipyramids were dispersed in CTAB solution $(1.5 \mathrm{~mL}, 1 \mathrm{mM})$ to be stored. 
Synthesis of Au NDBs: To prepare the nanodumbbells (bipyramids with spheres in the apexes), CTAC solution $(1.8 \mathrm{~mL}, 0.1 \mathrm{M})$ was mixed under stirring at $30{ }^{\circ} \mathrm{C}$ with the sequentially addition of CTAB solution $(20 \mu \mathrm{L}, 0.1 \mathrm{M}), \mathrm{HAuCl}_{4}(100 \mu \mathrm{L}, 0.002 \mathrm{M}), \mathrm{AgNO}_{3}(20 \mu \mathrm{L}, 0.002$ $\mathrm{M}), \mathrm{HCl}(20 \mu \mathrm{L}, 1 \mathrm{~N})$ and $\mathrm{AA}(40 \mu \mathrm{L}, 0.02 \mathrm{M})$, the colloidal solution was kept stirring for 5 min. Finally, purified bipyramid solutions in $10 \mathrm{mM} \mathrm{CTAB}(200 \mu \mathrm{L})$ was added and kept under stirring for two hours. The resulting solution was centrifuged at $10000 \mathrm{rpm}$ for $8 \mathrm{~min}$ and washed with CTAB $(1 \mathrm{mM})$, repeated twice, then dispersed in CTAB $(1 \mathrm{mM})$ for further characterization.

Purification of bipyramids and dumbbells was performed by several centrifugation cycles at $4000 \mathrm{rpm}$ in ultrapure water and mixtures of increasing ethanol proportion. The as-obtained Au NBPs and Au NDBs can be dispersed in water or ethanol.

Au NBPs ligand exchange: Ligand exchange process, to replace CTAB from the surface of Au NBPs with dodecanethiol, is based on aqueous-to-organic phase transfer, according to a previous method. ${ }^{[43]}$ The first step in the exchange reaction process was mixing the CTAB stabilized Au NBPs in aqueous solution with an excess of 1-dodecanethiol on the top of the solution, followed by the addition of acetone. The Au NBPs were extracted into the dodecanethiol phase after the mixture reaction was shake vigorously for 1 minute and left undisturbed until the phase separation occurred. After the procedure, it can be observed that the aqueous phase turned colorless, demonstrating the effectiveness of the proposed approach.

Au NBPs and Pt deposition: The as-prepared Au NBPs $(100 \mu \mathrm{L})$ in CTAB $(1 \mathrm{mM})$ were mixed with $\mathrm{CTAB}$ solution $(900 \mu \mathrm{L}, 0.1 \mathrm{M}), \mathrm{AgNO}_{3}$ aqueous solution $(10 \mu \mathrm{L}, 0.01 \mathrm{M})$, ascorbic acid solution $(8 \mu \mathrm{L}, 0.02 \mathrm{M})$ and $\mathrm{H}_{2} \mathrm{PtCl}_{6}$ aqueous solution $(70 \mu \mathrm{L})$ of different 
concentrations ranging from $10^{-6}$ to $10^{-4} \mathrm{M}$. The mixture was heated at $80{ }^{\circ} \mathrm{C}$ for 40 minutes under magnetic stirring.

Thermal experiments: For the thermal stability study of bipyramids, the samples were seeded on molybdenum TEM grids. Ex-situ thermal analysis was performed by heating the samples on the grids in an Evaporator-Intercovamex TE12 (High Vacuum System) at 200 and $400{ }^{\circ} \mathrm{C}$. In-situ heating experiments were performed inside the STEM Microscope by thermalizing the JEOL 21130 heating holder at $94{ }^{\circ} \mathrm{C}$.

Characterization of the obtained Au NBPs: Au Nanoparticles were characterized employing a Hitachi 5500 Field Emission Microscope operating at $30 \mathrm{kV}$, high-resolution Scanning Electron Microscopy (SEM) imaging and Energy Dispersive X-Ray Spectroscopy (EDS). High Resolution TEM imaging were taken with HRTEM JEOL 2010 and STEM imaging was carried out in a JEOL-ARM200F aberration-corrected microscope operating at $200 \mathrm{kV}$ in Scanning Transmission Electron Microscopy mode (STEM), coupled with High Angle Annular Dark Field (HAADF) detector and Energy Dispersive X-Ray Spectroscopy (EDS) analysis. Optical characterization was carried out in a Thermo Scientific Evolution 220 UVVis spectrophotometer. For the strain distribution in the interface of Au/Pt bipyramid was used geometric phase analysis (GPA).

\section{Conflict of Interest:}

The authors declare no conflict of interest.

\section{Acknowledgements}

The Welch Foundation supported this work through Grant No.: AX-1615-20170325. The authors also thank the National Council for Science and Technology (CONACYT), Mexico, for the support provided through the Abroad Postdoctoral Scholarship Program, and to the Kleberg Advanced Microscopy Center for the microscopy facilities. Also, Maria Fernanda Torresan thanks Fulbright Commission, Education, and Sports Argentinian Ministry and 
National Council for Science and Technology (CONICET), Argentina for the founds provided for the Exchange Visitor Program.

\section{References}

[1] M. Brust, C.J. Kiely, Colloids Surf. A Physicochem. Eng. Asp. 2002, 202, 175.

[2] C.M. Cobley, J. Chen, E.C. Cho, L. V Wang, Y. Xia, Chem. Mater. 2011, $40,44$.

[3] M. Hu, J. Chen, Z. Li, L. Au, G. V Hartland, X. Li, M. Marquez, Y. Xia, Chem. Soc. Rev. 2006, 35, 1084.

[4] Z. Liu, X. Zhang, L. Yang, L. Ren, D. Wong, J. Ye, Natl. Sci. Rev. 2017, 4, 761.

[5] S.D. Perrault, W.C.W. Chan, J. Am. Chem. Soc. Commun. 2009, 131, 17042.

[6] L.O. Brown, J.E. Hutchison, J. Am. Chem. Soc. 1999, 121, 882.

[7] S. Link, M.A. El-Sayed, J. Phys. Chem. B. 1999, 103, 4212.

[8] A. Genç, J. Patarroyo, J. Sancho-parramon, N.G. Bastús, V. Puntes, Nanophotonics 2017, 6, 193.

[9] B. Nikoobakht, M.A. El-sayed, Langmuir 2001, 17, 6368.

[10] M. Tsai, S.G. Chang, F. Cheng, V. Shanmugam, Y. Cheng, ACS Chem. Neurosci. 2013, 7, 5330.

[11] M. Liu, P. Guyot-Sionnest, J. Phys. Chem. B., 2005, 109, 22192.

[12] J.-H. Lee, K.J. Gibson, G. Chen, Y. Weizmann, Nat. Commun. 2015, 6, 7571.

[13] B.I.N. Wu, A. Heidelberg, J.J. Boland, Nat. Mater. 2005, 4, 525.

[14] S. Gong, W. Schwalb, Y. Wang, Y. Chen, Y. Tang, J. Si, B. Shirinzadeh, W. Cheng, Nat.

Commun. 2014, 5, 1.

[15] E.S. Shibu, N. Varkentina, L. Cognet, B. Lounis, Adv. Sci. 2017, 4, 1.

[16] S. Yung Lee, Y. Han, J. Wook Hong, J. Won Ha, Nanoscale 2017, 9, 12060.

[17] J. Burgin, M. Liu, P. Guyot-Sionnest, J. Phys. Chem. C. 2008, 112, 19278. 
[18] S.M.E. Peters, M.A. Verheijen, M.W.J. Prins, P. Zijlstra, Nanotechnology 2015, 27, 24001.

[19] S. Lee, K.M. Mayer, J.H. Hafner, Anal. Chem. 2009, 81, 4450.

[20] L. Zhou, Z. Liu, H. Zhang, S. Cheng, L.-J. Fan, W. Ma, Nanoscale 2014, 6, 12971.

[21] A. Lombardi, M. Loumaigne, A. Crut, P. Maioli, N. Del Fatti, F. Valle, Langmuir 2012, $28,9027$.

[22] X. Zheng, Y. Chen, Y. Chen, N. Bi, H. Qi, M. Qin, D. Song, H. Zhang, Y. Tian, J. Raman Spectrosc. 2012, 43, 1374.

[23] A. Caragheorgheopol, V. Chechik, Phys. Chem. Chem. Phys. 2008, 10, 5029.

[24] Y. Chen, Y. Xianyu, X. Jiang, Acc. Chem. Res. 2017, 50, 310.

[25] M. Moskovits, B. Vlčková, J. Chem. Phys. 2002, 116, 10435.

[26] E.C. Cho, L. Au, Q. Zhang, Y. Xia, Small. 2010, 6, 517.

[27] R. A. Sperling, W. J. Parak, Phil. Trans. R. Soc. 2010, 368, 1333.

[28] Z. Zhong, K.B. Male, J.H.T. Wong, J. Anal. Lett. 2003, 36, 3097.

[29] F. Fan, D. Liu, Y. Wu, S. Duan, Z. Xie, Z. Jiang, J. Am. Chem. Soc. 2008, 130, 6949.

[30] I. Park, K. Lee, J. Choi, H.Park, Y. Sung, J. Phys. Chem. C. 2007, 111, 19126.

[31] Z.Y. Bao, D.Y. Lei, R. Jiang, X. Liu, J. Dai, J. Wang, H. L. W. Chan, Y. H. Tsang, Nanoscale 2014, 6, 9063.

[32] C. Tan, Y. Sun, J. Zheng, D. Wang, Z. Li, H. Zeng, L. Guo, L. Jing, L. Jiang, Nat. Sci. Rep. 2017, 7, 6347.

[33] W. Xie, C. Herrmann, K. Kömpe, M. Haase, S. Schlücker, J. Am. Chem. Soc. 2011, 133, 19302.

[34] S. Link, Z.L. Wang, J. Phys. Chem. 1999, 103, 3529.

[35] M. Hosseini, T. Barakat, R. Cousin, A. Aboukaïs, B. Su, G. De Weireld, Appl. Catal. B. 2012, 112, 218. 
[36] Y. Ma, X. Zhu, S. Xu, G, He, L. Yao, N. Hu, Y. Su, J. Feng, Y. Zhang, Z. Yang, App. Catal. B, 2018, 234, 26.

[37] M. Grzelczak, J. Pérez-Juste, F. J. García de Abajo, L. M. Liz-Marzán, J. Phys. Chem. C, 2007, 111, 6183 .

[38] M.M. Maye, W. Zheng, F.L. Leibowitz, N.K. Ly, C.-J. Zhong, Langmuir 2000, 16, 490. [39] T. Teranishi, S. Hasegawa, T. Shimizu, M. Miyake, Adv. Mater. 2001, 13, 1699.

[40] B.J.Y. Tan, C.H. Sow, T.S. Koh, K.C. Chin, A.T.S. Wee, C.K. Ong, J. Phys. Chem. B. 2005, 109, 11100 .

[41] C. Y. Yang, M. José -Yacaman, K. Heinemann, J. Cryst. Growth 1979, 42, 283.

[42] Y. N. Wen, J. M. Zhang, Solid State Comm. 2007, 144, 163.

[43] L. Vitos, A. V. Ruban, A. V. Skriver, H. L. Kollar, Surf. Sci. 1998, 411, 186.

[44] R. Mendoza-Cruz, L. Bazan-Díaz, J. J. Velázquez-Salazar, J. E. Samaniego-Benitez, F. M. Asencio-Aguirre, R, Herrera-Becerra, M. José-Yacamán, G. Guisbiers, Nanoscale 2017, 9, 9267.

[45] X. Kou, W. Ni, C.K. Tsung, K. Chan, H.Q. Lin, G.D. Stucky, J. Wang, Small 2007, 3, 2103.

[46] R. Long, S. Zhou, B.J. Wiley, Y. Xiong, Chem. Soc. Rev. 2014, 43, 6288.

[47] Z. Zhang, M. Lin, RSC Adv. 2014, 4, 17760.

[48]A. Wijaya, K. Hamad-schifferli, Langmuir 2008, 24, 9966.

[49] B. Varnholt, P. Oulevey, S. Luber, C. Kumara, A. Dass, T. Bürgi, J. Phys. Chem. C. 2014, 118, 9604.

[50] D. Jaque, L. Martínez Maestro, B. del Rosa, P. Haro-Gonzalez, A. Benayas, J.L. Plaza, E. Martín Rodriguez, J. García-Solé, Nanoscale 2014, 6, 9494.

[51] L. Bazán-Díaz, R. Mendoza-Cruz, J.J. Velázquez-Salazar, G. Plascencia-Villa, D. Romeu, J. Reyes-Gasga, R. Herrera-Becerra, M. José-Yacaman, G. Guisbiers, Nanoscale 2015, 7, 20734. 
[52] C.F. Landes, S. Link, M.B. Mohamed, B. Nikoobakht, M.A. El-Sayed, Pure Appl. Chem. 2002, 74, 1675.

[53] D.J. Lavrich, S.M. Wetterer, S.L. Bernasek, G. Scoles, J. Phys. Chem. B., 1998, 102, 3456.

[54] W.H. Li, S.Y. Wu, C.C. Yang, F.C. Tsao, S.K. Lai, K.C. Lee, Phys. Rev. Lett. 2002, 89, 135504.

[55] T. Burgi, Nanoscale 2015, 7, 15553.

[56] P. Hernandez-Fernandez, S. Rojas, P. Ocon, J. Gomez de la Fuente, J. San Fabian, J.

Sanza, M. A. Peña, J. García-García, P. Terreros, J. Fierro, J. Phys. Chem. C. 2007, 111, 2913.

[57] C.A. García-Negrete, B.R. Knappett, F.P. Schmidt, T.C. Rojas, A.E.H. Wheatley, F.

Hofer, A. Fernández, RSC Adv. 2015, 5, 55262.

[58] A. Baskaran, P. Smereka, J. Appl. Phys. 2012, 111, 044321.

[59] D.A. Smith, G. Shiflet, Mater. Sci. Eng. 1987, 86, 67.

[60] R. Koch, D. Hu, A.K. Das, Phys. Rev. Lett. 2005, 94, 146101.

[61] C. Pecharromán, J. Pérez-Juste, G. Mata-Osoro, L. Liz-Marzán, P. Mulvaney Phys. Rev.

B 2008, 77, 035418

[62] M. J. Hytch, E. Snoeck, R. Kilaas, Ultramicroscopy 1998, 74, 131 